\title{
Estimation of the Thyroid Secondary Cancer Risk on the Patient of Standard Breast External Beam Radiotherapy
}

\begin{abstract}
Background: The purpose of this study was to estimate the secondary cancer risk of thyroid in standard radiotherapy methods which are commonly used for breast cancer patients. Methods: A total of 64 breast cancer patients (their age range was around 50 years old) who referred to Seyed-Al-Shohada hospital (Isfahan, Iran) were included in this study. The radiotherapy of the mentioned patients was performed using 6-MV photon beams. Dose measurements were also done using thermoluminescent dosimeters. Calculation of the risk of developing secondary cancer in thyroid was done using the Biological Effects of Ionizing Radiation Committee VII and recommended quantity of the International Radiation Protection Commission, excess relative risk. Results: The mean radiation dose to thyroid for the tangential beams, tangential field with supraclavicular (SC) field, and also a tangential field with SC field in modified radical mastectomy (MRM) were $0.883 \pm 0.472,1.512 \pm 0.365$, and $1.587 \pm 0.37$, respectively. The risk of developing secondary thyroid cancer over a period of 5 years after breast cancer therapy in the tangential, tangential with a SC field, and also tangential beam with SC field in MRM were 9.974 $\pm 4.318,17.891 \pm 0.365$, and $18.783 \pm 4.384$, respectively. The mean of the measured thyroid doses in patients treated with tangent fields was significantly lower than the patients under the irradiation of the tangent fields with SC field $(P<0.001)$. Conclusions: Using radiation protection equipment is suggested for breast cancer patients who treated with the studied radiotherapy methods.
\end{abstract}

Keywords: Breast cancer, second cancer risk, thyroid, TLD, radiotherapy

\section{Introduction}

Breast cancer is one of the most common malignancies, which are more prevalent in the developing countries. ${ }^{[1]}$ The annual rate of incidence of breast cancer risk is approximately 1 million worldwide. ${ }^{[1-3]}$ Radiation therapy is one of the most common approaches to treat breast cancer treatment. ${ }^{[4]}$ In external radiation therapy, healthy tissue outside the radiation field is inevitably exposed to out of field radiation. The imposed dose in this region is called peripheral dose (PD). ${ }^{[5,6]}$ The PD sources are leakage and scattered radiation from the linac head and scattered radiation from the irradiated volume of patient. ${ }^{[7,8]}$ The goal of the treatment planning process is to deliver prescribed dose to the target volume and to limit the organ at risk dose. ${ }^{[9,10]}$ The absorbed dose of out of field tissues is not respected in treatment planning because of the limitation of treatment planning

This is an open access journal, and articles are distributed under the terms of the Creative Commons Attribution-NonCommercial-ShareAlike 4.0 License, which allows others to remix, tweak, and build upon the work non-commercially, as long as appropriate credit is given and the new creations are licensed under the identical terms.

For reprints contact: reprints@medknow.com systems (TPS) in the calculation of the accurate dose in out of field region and also the lack of tomographic images of this region in routine imaging prescription. The ability of several TPS inaccurate dose calculation is limited to $3 \mathrm{~cm}$ out of field region. ${ }^{[1]}$ Therefore, the estimation of the risk of developing secondary cancers after radiotherapy was the goal of several studies. ${ }^{[12]}$ The risk of developing secondary cancer in patients who undergone radiation therapy has long been known. ${ }^{[8]}$ Several studies have shown that women with breast cancer have an additional risk for other cancers such as thyroid cancer. ${ }^{[12-14]}$ Many studies evaluated the risk of secondary cancer in radiotherapy of breast cancer patients and also a great variety can be seen in their methodology (measurement ${ }^{[15]}$ or calculation ${ }^{[16]}$, dosimetry mediums (phantom $^{[17,18]}$ or patient ${ }^{[19]}$ ), dosimeters (thermoluminescent dosimeter [TLD], metal-oxide-semiconductor field-effect transistor or other types), ${ }^{[13,17,20]}$ treatment techniques (standard or complex), ${ }^{[17,18]}$ type

\footnotetext{
How to cite this article: Momeni Z, Tavakoli MB, Atarod M. Estimation of the thyroid secondary cancer risk on the patient of standard breast external beam radiotherapy. J Med Sign Sens 2018;XX:XX-XX.

Received: March, 2018. Accepted: June, 2018.
}

\section{Zeinab Momeni' ${ }^{1}$, Mohammad Bagher Tavakoli ${ }^{1}$, Maryam Atarod ${ }^{2}$}

${ }^{1}$ Department of Medical Physics, School of Medicine, Isfahan University of Medical Sciences, Isfahan, ${ }^{2}$ Department of Medical Physics, School of Medicine, Shahrekord University of Medical Sciences, Shahrekord, Iran

Address for correspondence: Dr. Mohammad Bagher Tavakoli,

Department of Medical Physics, School of Medicine, Isfahan

University of Medical Sciences, Isfahan, Iran.

E-mail:mbtavakoli@mui.ac.ir

Website: www.jmss.mui.ac.ir DOI: 10.4103/jmss.JMSS_12_18 
of treatment machines, reported quantity (medium dose, equivalent dose, and skin dose), ${ }^{[20-22]}$ and the risk models (International Radiation Protection Commission [ICRP] or biological effects of ionizing radiation [BEIR]). ${ }^{[18,23]}$

The results of many studies have indicated that this risk cannot be ignored. ${ }^{[15]}$ A number of studies demonstrated different radiation dose for thyroid in the mentioned patients who were treated with different methods. The results of an in vivo study on 23 patients, who underwent breast radiotherapy using tangential and supraclavicular (SC) fields, shown the thyroid dose were 2 and $8 \%$ of the prescribed dose, respectively. ${ }^{[13]}$ In another study, the skin entrance dose (SED) of thyroid following breast radiotherapy was $7 \%$ of the prescribed dose for SC field, which was more than thyroid's tolerance. ${ }^{[17]}$ In another study on 69 patients, the mean reported thyroid doses were $3.7 \%$ of the delivered dose measured by the TLD. The results of this study indicate a significant risk of thyroid cancer after breast radiotherapy and the need for thyroid protection especially for young patients. ${ }^{[21]}$ Thyroid absorbed dose in out of breast radiotherapy fields depends on the field size and distance from field border. Therefore, thyroid dose differs in radiotherapy with different treatment plans and treatment machines. The purpose of this study was to measure the absorbed dose of thyroid and estimate the risk of its secondary cancer in breast cancer patients who treated with different radiotherapy techniques including the following: tangential beams, tangential with SC fields, and also a tangential field with SC field in modified radical mastectomy (MRM), using 6-MV photon beams of an ELECTA linear accelerator.

\section{Materials and Methods}

A total of 64 breast cancer patients who referred to Seyed-Al-Shohada Hospital (Isfahan, Iran) were included in this study. The TiGRT (Linatech company) TPS was used to design the treatment plans. The planning target volume and the organs at risk were specified by a radiation oncologist. The prescribed dose of tumor for each patient was 50 Gy in 25 fractions. The standard treatment plans of the selected patients classified in two groups as follows: (1) whole breast irradiation with two tangential field techniques (Tan) and (2) two tangential field plus two SC lymph node fields technique (Tan + SCL). About 64 evaluated patients, 29 patients with mean age of $42.34 \pm 6.48$ were treated with Tan plan, 35 patients with a mean age of $43.94 \pm 5.63$ and also 34 mastectomy patients a mean age of $42.18 \pm 5.52$ were treated with Tan + SCL plan. Summary of the treatment plans characteristics is shown in Table 1. The selected patients were chosen according to the following criteria: the patients younger than 50 were selected for the study, because of the longer lifespan of younger patients. ${ }^{[24-26]}$ Considering the high dependence of PD on the distance from the radiation field, ${ }^{[22]}$ another criterion for patient selection was the distance between the thyroid and the irradiated breast, which was determined using computed tomographic images of the patients. The distance between the upper limit of the breast and the lower limit of the thyroid gland was measured as the thyroid distance from the breast. Dose measurements were performed using LiF Thermoluminescence dosimeters LiF (TLD-100). Kry has reported that TLD-100 is well suited for measuring out-of-field dose at $6 \mathrm{MV} .^{[27]}$ TLDs were read out using a SOLARO-2A Model TLD reader (NE company) according to the protocol suggested by manufacturer. TLDs were calibrated with 6-MV beams of ELECTA linac. The thyroid dose measurements were performed for each patient at three locations specified by a radiation oncologist. According to the Schneider et al., the average dose can be considered as an average thyroid dose. ${ }^{[23]}$ Therefore, three TLDs were located in the middle of the two right and left lobes and in the middle of the thyroid. The average of these three dose values was considered as thyroid dose for risk calculation.

To calculate the radiation-induced second thyroid cancer, the BEIR committee cancer incidence model was used. The BEIR VII model provides organ-specific parameters for each sex and includes a parameter relating cancer incidence risk with age at exposure and attained age. Equation (1) is the BEIR VII model for both excess relative risk (ERR) and excess absolute risk. In this equation, $\mathrm{D}$ is radiation absorbed dose; $\beta_{S}, \gamma$, and $\eta$ are organ-specific parameters for each sex; e is age at exposure; $\mathrm{e}^{*}=(\mathrm{e}-30) / 10$ for $\mathrm{e}<30$ and 0 for e $>30$ years; and a is the attained age.

The thyroid-specific parameters of the model $\left(\beta_{S}, \gamma\right.$, and $\left.\eta\right)$ are presented in Table 2.

$\mathrm{ERR}$ and $\mathrm{EAR}=D \beta$ s $\exp \left(\gamma e^{*}\right)\left(\frac{a}{60}\right)^{\eta}$

The ERR of secondary thyroid cancer for 5, 10, and 15 years after breast radiotherapy was calculated using the

\begin{tabular}{lccc}
\hline $\begin{array}{l}\text { Table 1: Summary of the treatment plans characteristics } \\
\text { used for breast radiotherapy of evaluated patients }\end{array}$ \\
$\begin{array}{l}\text { Treatment } \\
\text { planning }\end{array}$ & Field size & $\begin{array}{c}\text { Angle of gantry } \\
\text { (degree) }\end{array}$ & $\begin{array}{c}\text { Prescribed } \\
\text { dose (cGy) }\end{array}$ \\
\hline Tan & & & \\
Middle field & $20.7 \times 15.3$ & 292.9 & 2500 \\
Lateral field & $20.1 \times 15.5$ & 121.8 & 2500 \\
Tan + SCL & & & \\
Middle field & $16.8 \times 11.8$ & 49 & 2500 \\
Lateral field & $17.4 \times 11.2$ & 220.3 & 2500 \\
Anterior field & $14.6 \times 5.8$ & 3.9 & 3750 \\
Posterior field & $14.6 \times 7.0$ & 183.9 & 1250 \\
\hline
\end{tabular}

$\mathrm{SCL}$ - Supraclavicular lymph node

Table 2: Thyroid-specific parameters of biological effected of ionizing radiation committee VII risk model (1)

\begin{tabular}{lcccc}
\hline Location of cancer & $\boldsymbol{\gamma}$ & $\boldsymbol{\beta}_{\mathbf{M}}$ & $\boldsymbol{\beta}_{\mathbf{F}}$ & $\boldsymbol{\eta}$ \\
\hline Thyroid & -0.3 & 0.45 & 0.27 & -2.8 \\
\hline
\end{tabular}


measured average dose values of thyroid in each breast treatment plans and equation. ${ }^{[1]}$

Statistical analysis in this study was performed using SPSS (ver 20) software. The statistical tests selection was performed based on the type of our variables and their probable dependence. The one-way ANOVA test was applied on age-related data and thyroid doses in three different thyroid lobes. The post hoc test (Tukey HSD) was used to compare the average dose values of three different lobes of the thyroid. To find any dependency of mean thyroid dose on the side of the irradiated breast, the independent $t$-test is used.

\section{Results}

Table 3 indicates the mean thyroid doses in the evaluated patients who were included in this study. Table 3 also illustrates the results of the thyroid dose measurements for the mastectomy patients.

Table 3, compares the measured radiation dose for thyroid among left and right breast cancer patients. The percentage of patients with right breast cancer with Tan and Tan+SCL plans were $58.6 \%$ and $62.9 \%$, respectively. The number of mastectomy patients with right and left breast cancer was equal in this study.

Table 4, indicates the dependent Variable: Relationship of mean measured dose of thyroid and treatment plan (Tan or Tan+SCL or MRM) (Tukey HSD).

The results of average thyroid dose values against the distance of thyroid from SC field, is illustrated in Figure 1. Because of the field size dependence of PD, the thyroid dose values of irradiated patients with $10 \times 10 \mathrm{~cm}^{2}$ equivalent field size, are shown in this Figure 2.

Figure 3 shows the ERR data of developing secondary thyroid cancer following breast cancer radiotherapy with different treatments.

\section{Discussion}

The aim of this study was to measure thyroid doses and to estimate the risk of developing secondary cancers of the thyroid after breast cancer radiation therapy. The thyroid is a radiosensitive organ, and there are a lot of evidence for the incidence of radiation-induced cancer in radiotherapy patients. According to the Radiation Therapy Oncology Group report, to reduce thyroid damage, the maximum thyroid dose should be kept below 3\% of the prescribed dose. ${ }^{[15,17]}$ For this reason, our study aimed to evaluate the thyroid risk in breast cancer patients who underwent radiotherapy in Seyed-Al-Shohada Hospital at Isfahan.

Many studies have been conducted on the incidence of thyroid secondary cancer in breast cancer when is treated with radiation. Among several studies aimed to measure the thyroid dose during breast cancer radiotherapy, those with

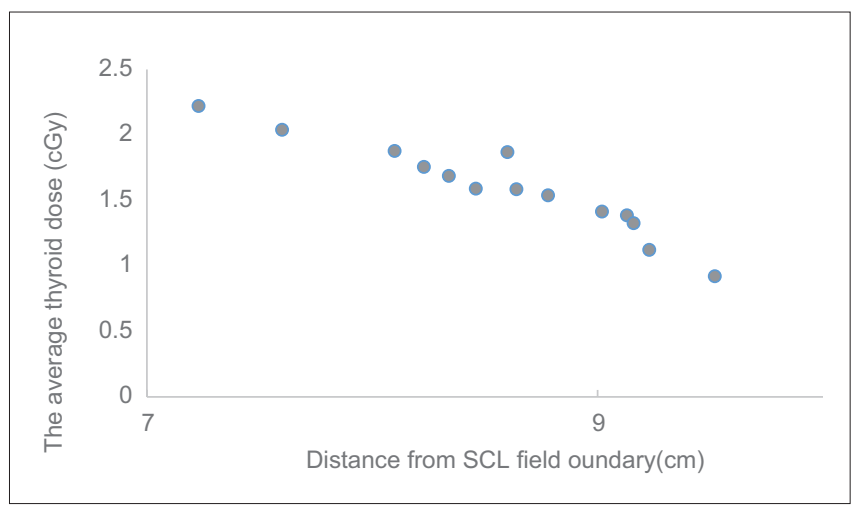

Figure 1: Thyroid dose as a function of the distance of thyroid from the supraclavicular field edge in breast radiotherapy with 200 cGy fraction size

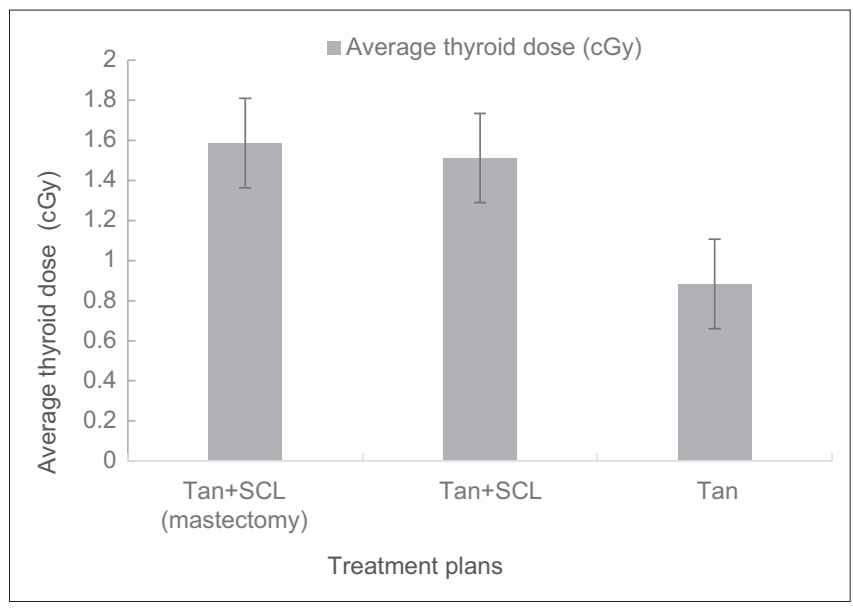

Figure 2: Mean thyroid dose (cGy) from breast radiotherapy (in one 200 cGy fraction) with different techniques

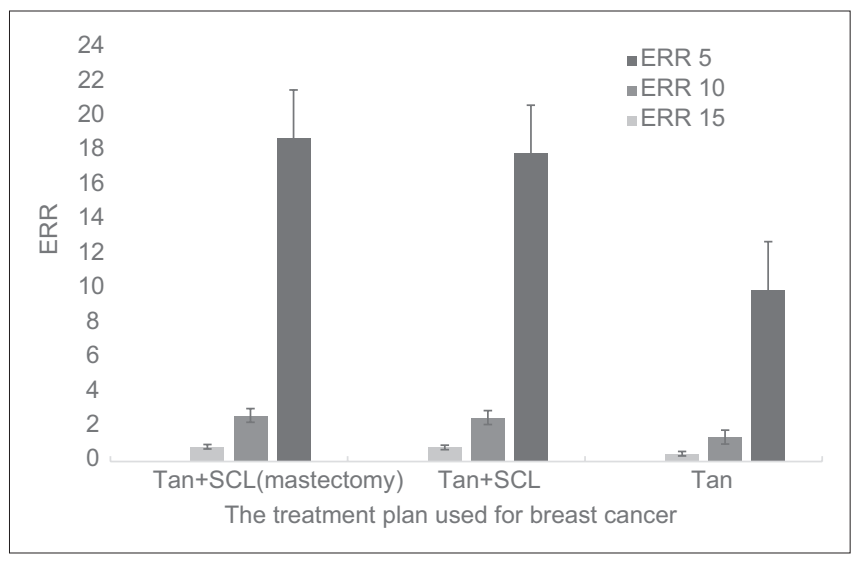

Figure 3: Excess relative risk of secondary thyroid cancer following breast radiation therapy with different plans

approximately similar treatment plans and methodology were selected for comparison. Vlachopoulou et al. reported thyroid dose, using metal-oxide-semiconductor field-effect transistor, $8 \% \pm 2 \%$ and $2 \% \pm 0.8 \%$ of the prescribed dose, following three-dimensional conformal radiotherapy of breast with and without SCL field, respectively. The relevant risk of secondary thyroid cancer of obtained 


\begin{tabular}{|c|c|c|c|c|}
\hline Treatment plan & The side of the treated breast & The range of thyroid doses & Thyroid mean dose \pm SD & $P$ \\
\hline \multirow[t]{2}{*}{ Tan fields } & Right & $0.286-1.506$ & $0.85 \pm 0.337$ & 0.549 \\
\hline & Left & $0.39-1.526$ & $0.93 \pm 0.369$ & \\
\hline \multirow[t]{2}{*}{ Tan + SCL fields } & Right & $0.566-2.22$ & $1.542 \pm 0.39$ & 0.536 \\
\hline & Left & $0.985-2.038$ & $1.462 \pm 0.328$ & \\
\hline \multirow[t]{2}{*}{ Tan + SCL fields (mastectomy) } & Right & $0.886-2.26$ & $1.629 \pm 0.336$ & 0.524 \\
\hline & Left & $0.701-2.25$ & $1.546 \pm 0.407$ & \\
\hline
\end{tabular}

SCL - Supraclavicular lymph node; SD - Standard deviation

thyroid dose is calculated using the ICRP 103 risk model $1 \%$ and $0.3 \%$, respectively. ${ }^{[13]}$ Farhood et al. reported SED 7\% of the prescribed dose in one fraction in patients undergoing breast treating with Tan and SC plans using TLD. ${ }^{[17]}$ In another study, the mean reported thyroid dose in 69 patients undergoing radiation therapy for breast cancer using TLD was $3.7 \%$ of the prescribed dose, which is consistent with $3.02 \%$ measured in our study. ${ }^{[21]}$ In a study by Donovan et al., thyroid dose in an anthropomorphic phantom under breast radiotherapy with Tan plan using TLD was reported $0.3 \%$ of the delivered dose against $1.76 \%$ measured in our study. ${ }^{[16]}$ Our findings verify the dependency of the thyroid dose on the distance of the thyroid from the field edge. The highest and lowest thyroid dose values were measured $3.17 \mathrm{cGy}$ in SC field and 0.29 cGy in Tan field, respectively. The major contribution of SCL fields in thyroid dose is due to the less distance of the thyroid to SC field edge. The dose near the field edge is found higher [Figure 2] which may be due to the penumbra of the primary beam, ${ }^{[28]}$ the steep variation of thyroid dose with distance from the field edge is related to rapid attenuation of scattered radiation within the patient. ${ }^{[29]}$ By performing one-way ANOVA on age-related data and thyroid doses in three different thyroid lobes, we concluded that the mean age of the patients was the same in three evaluated groups $(P=0.394)$. The average dose values of three different lobes of the thyroid were significantly different between three groups $(P<0.001)$. The results of the post hoc test (Tukey HSD) show that the mean measured dose of thyroid in patients treated with Tan plan was significantly lower than in patients undergoing Tan + SCL plans $(P<0.001)$ which is in confirmation with Vlachopoulou et al. finding. ${ }^{[13]}$ The proximity of the thyroid to the SC fields and high dependency of PD to distance from field edge could be the cause of this difference. Comparison of the average thyroid dose in patients undergoing breast-saving and mastectomy surgery against who had treated with the same Tan + SCL plan, does not show any significant difference $(P=0.667)$.

Independent $t$-test results indicate that the measured mean thyroid dose is independent on the side of the irradiated breast $(P=0.53)$. According to BEIR VII and ICRP reports, the radiation-induced secondary cancer more likely incidence during $<10$ years after radiotherapy.
Table 4: Comparision of mean measured dose of thyroid among subjects group

\begin{tabular}{llcc}
\hline Group (I) & Group (J) & Mean difference (I-J) & $\boldsymbol{P}$ \\
\hline Tan & Supra & -0.6295 & 0.001 \\
\multirow{2}{*}{ Supra } & MRM & -0.7045 & 0.001 \\
& Tan & 0.6295 & 0.001 \\
& MRM & -0.0749 & 0.667 \\
\hline
\end{tabular}

$\overline{\text { SCL - Supraclavicular lymph node; MRM - Modified radical }}$ mastectomy

\section{Conclusions}

In this study, the imposed dose of thyroid and the risk of its secondary cancer in breast cancer patients who treated with tangential beams, tangential with SC field, and also tangential field with SC field in MRM plans were evaluated.

Based on the results of this study, to reduce the risk of secondary thyroid cancer in a patient treated with radiotherapy technique for breast cancer, protection of the thyroid gland is necessary.

\section{Acknowledgment}

This article is based on the master's research project with number 396476, which is the financial and credit source of the Isfahan University of Medical Sciences. The authors are keen to thank the staff members of the Seyed al-Shohada Hospital (Isfahan, Iran) who participated in the project.

Financial support and sponsorship

None.

\section{Conflicts of interest}

There are no conflicts of interest.

\section{References}

1. Halperin EC, Perez CA, Brady LW. Perez and Brady's principles and practice of radiation oncology: Lippincott Williams \& Wilkins; 2008;1168-1169:1246-1247.

2. Jemal A, Bray F, Center MM, Ferlay J, Ward E, Forman D, et al. Global cancer statistics. CA Cancer J Clin 2011;61:69-90.

3. Prabhakar R, Rath GK, Julka PK, Ganesh T, Joshi RC, Manoharan $\mathrm{N}$, et al. Breast dose heterogeneity in CT-based radiotherapy treatment planning. J Med Phys 2008;33:43-8.

4. Khan FM. Treatment Planning in Radiation Oncology. $2^{\text {nd }}$ ed. 
Philadelphia: Lippincott Williams \& Wilkins; 2007.148-50.

5. Jia MX, Zhang X, Yin C, Feng G, Li N, Gao S, et al. Peripheral dose measurements in cervical cancer radiotherapy: A comparison of volumetric modulated arc therapy and step-and-shoot IMRT techniques. Radiat Oncol 2014;9:61.

6. Sánchez-Nieto B, El-Far R, Irazola L, Romero-Expósito M, Lagares J, Mateo J, et al. Analytical model for photon peripheral dose estimation in radiotherapy treatments. Biomed Phys Eng Expr 2015;1:045205.

7. Chofor N, Harder D, Willborn KC, Poppe B. Internal scatter, the unavoidable major component of the peripheral dose in photon-beam radiotherapy. Phys Med Biol 2012;57:1733-43.

8. Xu XG, Bednarz B, Paganetti H. A review of dosimetry studies on external-beam radiation treatment with respect to second cancer induction. Phys Med Biol 2008;53:R193-241.

9. Li T, Ao EC, Lambert B, Brans B, Vandenberghe S, Mok GS, et al. Quantitative imaging for targeted radionuclide therapy dosimetry - Technical review. Theranostics 2017;7:4551-65.

10. Stoval 1, Blackwell C, Cundiff J. Fetal dose from radiotherapy with photon beams: Report of AAPM Radiation Therapy Committee Task Group No. 36. Med Phys 1995:22:63-82.

11. Howell RM, Scarboro SB, Taddei PJ, Krishnan S, Kry SF, Newhauser WD, et al. Methodology for determining doses to in-field, out-of-field and partially in-field organs for late effects studies in photon radiotherapy. Phys Med Biol 2010;55:7009-23.

12. Schneider U. Modeling the risk of secondary malignancies after radiotherapy. Genes (Basel) 2011;2:1033-49.

13. Vlachopoulou V, Malatara G, Delis H, Kardamakis D, Panayiotakis G. Estimation of the risk of secondary cancer in the thyroid gland and the breast outside the treated volume in patients undergoing brain, mediastinum and breast radiotherapy. Radiat Prot Dosimetry 2013;154:121-6.

14. Badrian H, Sheikhi M, Mirzabagherian A, Khalighinezhad N. Evaluation of the absorbed dose of the thyroid gland in conventional spiral and spiral computed tomography techniques. J Isfahan Dent Sch. 2012;8:143-50.

15. Lee B, Lee S, Sung J, Yoon M. Radiotherapy-induced secondary cancer risk for breast cancer: 3D conformal therapy versus IMRT versus VMAT. J Radiol Prot 2014;34:325-31.

16. Donovan EM, James H, Bonora M, Yarnold JR, Evans PM. Second cancer incidence risk estimates using BEIR VII models for standard and complex external beam radiotherapy for early breast cancer. Med Phys 2012;39:5814-24.

17. Farhood B, Bahreyni Toossi MT, Vosoughi H, Khademi S, Knaup C. Measurement of thyroid dose by TLD arising from radiotherapy of breast cancer patients from supraclavicular field. J Biomed Phys Eng 2016;6:147-56.

18. Han EY, Paudel N, Sung J, Yoon M, Chung WK, Kim DW, et al. Estimation of the risk of secondary malignancy arising from whole-breast irradiation: Comparison of five radiotherapy modalities, including tomoHDA. Oncotarget 2016;7:22960-9.

19. Gul A, Faaruq S, Abbasi NZ, Siddique T, Ali A, Shehzadi NN, et al. Estimation of absorbed dose to thyroid in patients treated with radiotherapy for various cancers. Radiat Prot Dosimetry 2013;156:37-41.

20. Tootell A, Szczepura K, Hogg P. An overview of measuring and modelling dose and risk from ionising radiation for medical exposures. Radiography 2014;20:323-32.

21. Sulieman A, Theodorou K, Kappas C. Entrance and peripheral doses measurements during radiotherapy. J Sci Technol 2011;12:20-7.

22. Mohsin NI, Zakaria A, Abdullah R, Wong MF. Peripheral dose measurement for $6 \mathrm{MV}$ photon beam. J Med Phys Biophys 2014;1:14-5.

23. Schneider U, Zwahlen D, Ross D, Kaser-Hotz B. Estimation of radiation-induced cancer from three-dimensional dose distributions: Concept of organ equivalent dose. Int J Radiat Oncol Biol Phys 2005;61:1510-5.

24. Mousavi SM, Montazeri A, Mohagheghi MA, Jarrahi AM, Harirchi I. [Breast Cancer in Iran: An Epidemiological Review (Persian)]. The Breast Journal 2007;13:383-391.

25. Khazaee-pool M, Majlessi F, Foroushani AR, Montazeri A, Nedjat S, Shojaeizadeh D, et al. Perception of breast cancer screening among Iranian women without experience of mammography: A qualitative study. Asian Pac J Cancer Prev 2014;15:3965-71.

26. Mousavi SM, Montazeri A, Mohagheghi MA, Jarrahi AM, Harirchi I, Najafi $M$, et al. Breast cancer in Iran: An epidemiological review. Breast J 2007;13:383-91.

27. Kry SF, Titt U, Followill D, Pönisch F, Vassiliev ON, White RA, et al. A monte carlo model for out-of-field dose calculation from high-energy photon therapy. Med Phys 2007;34:3489-99.

28. Uddin RM, Alam MJ, Ahmad G. Measurement of dose out side the irradiated volume by using locally fabricated water phantom. Bangladesh J Med Phys 2003;2:41-3.

29. Taylor ML, Kron T. Consideration of the radiation dose delivered away from the treatment field to patients in radiotherapy. J Med Phys 2011;36:59-71. 


\section{BIOGRAPHIES}

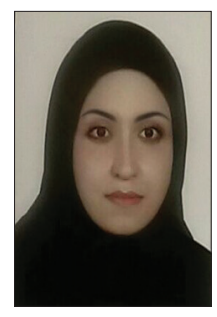

Zeinab Momeni, received the B.Sc. degree in radiology from Shahid Sadoughi University of Medical Sciences and Health Services, Yazd, Iran in 2013 and the M.Sc. in Medical Physics from University of Isfahan of Medical Sciences, Iran in 2018.

Email: zeinabmomeni1397@gmail.com

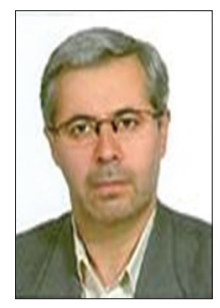

Mohammad Bagher Tavakoli, received the B.Sc. degree in Physics from Isfahan University, Iran in 1980 and the M.Sc. in Medical Physics from University of Leeds, England in 1987 and Ph.D in Medical Physics from University of Leeds, England in 1990 .

Appointments: Member of Isfahan University of Medical Sciences Trusty Board and Head of the Medical Physics and Medical Engineering department, from 2006 till now.

Email: mbtavakoli@mui.ac.ir

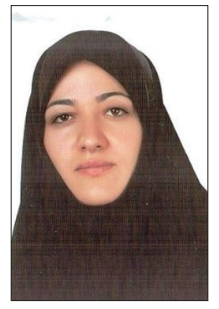

Maryam Atarod, received the B.Sc. degree in Atomic Physics from Isfahan University, Iran in 2000 and the M.Sc. in Medical Physics from Isfahan University of Medical Sciences, Iran in 2003 and Ph.D in Medical Physics from Isfahan University of Medical Sciences, Iran in 2013.

Current academic activities

1. Teaching medical and radiological Physics courses.

2. Research in radiotherapy, dosimetry and Monte Carlo simulations

Email:maryamatarod@yahoo.com 